\title{
Comparison of the EMG Activities in the Vastus Medialis Oblique and Vastus Lateralis Muscles During Hip Adduction and Terminal Knee Extension Exercise Protocols
}

\author{
Balogun, J.A., Broderick, K., Dolan-Aiello, M. \\ Correspondence \\ Professor Joseph A. Balogun, Dean, College of Health Sciences, Chicago State University, 9501 South King \\ Drive, BHS 607, Chicago, IL 60628, USA・Email: Jbalogun@csu.edu
}

\begin{abstract}
SUMMARY
The purpose of this study was to compare the electromyographic (EMG) activities in the vastus medialis oblique (VMO) and vastus lateralis (VL) muscles during two open chain exercises commonly used in the management of patellofemoral pain syndrome (PFPS).

Twenty-five (14 female and 11 male) healthy subjects participated in the study. The A 2 (testing condition) x 2 (exercise type) x 2 (gender) quasi-experimental design was employed. Using surface electrodes, the EMG activities in the VMO and the VL muscles during ten repetitions of hip adduction and terminal knee extension exercises were recorded. The order of presentation of the experimental conditions was randomized.

The three-way repeated ANOVA revealed no significant main effects for testing conditions, exercise type or gender, but the terminal knee extension exercise type $\mathrm{x}$ gender interaction effect was statistically significant $(\mathrm{F}=8.28, \mathrm{p}<.001)$. During the terminal knee extension exercise, the EMG activities in the VL muscle of the male subjects were higher $(\mathrm{p}<.05)$ than in the VMO muscle. For both genders, no significant difference was found in the EMG activities of both muscles during the hip adduction exercise.

Our results revealed that neither of the two exercise methods was more effective in activating the VMO or VL muscles. Further studies are needed to identify the most effective exercise protocol for strengthening the quadriceps femoris of patients with PFPS.
\end{abstract}

KEY WORDS: electromyography (EMG); quadriceps femoris; hip adduction and terminal knee extension exercises

\section{INTRODUCTION}

Chondromalacia patellae, also known as patellofemoral pain syndrome (PFPS), is a challenging musculoskeletal problem to manage (Grelsamer 2005, Jenkins and Honig, 2005). It frequently occurs in teenagers, manual labourers, and athletes and is characterized by wearing down, roughening, or softening of the cartilage under the patella. It is caused by overuse, injury, excess weight, and a patella that is not properly aligned (patellar tracking disorder), or changes under the patella (Schindler 2004, http://www.jgames.co.uk/title/Chondromalacia_patellae).

The main symptom of PFPS is pain in the front of the knee, especially when sitting with bent knees, squatting, jumping, or using the stairs (especially going down stairs). Other symptoms include occasional knee buckling, in which the knee suddenly and unexpectedly gives way and does not support the body weight; a catching, popping, or grinding sensation when walking or with knee movement (http://www.webmd.com/a-to-z-guides/patellofemoral-painsyndrome-topic-overview). Conservative treatments indicated in the management of PFPS are: nonprescription anti-inflammatory drugs and cryotherapy to relieve pain and swelling, and exercise therapy to stabilize the patella complex, increase flexibility and strengthen the quadriceps muscle (Hecox et al., 2006. http://www.medicinenet.com/ patellofemoral_syndrome/article.htm).

Various hypotheses have been offered in the extant literature on the aetiology of patellar tracking disorder associated with PFPS. The action of the vastus medialis oblique (VMO) muscle which counteracts the pull of the vastus lateralis (VL) muscle is considered to be critical in the maintenance of patellar stability. Some investigators believe PFPS is caused by differences in the timing during recruitment of the VMO and the VL muscles during gait 
(Voight and Wierder, 1991), while others hypothesize that an imbalance in the strength of the VMO and VL muscles is the culprit (Karst and Willet, 1995; Cerny, 1995; Hodges and Richardson, 1993; Hanten and Schulthies, 1990; Doucette and Child, 1996; Zakaria et al., 1997).

The effects of specific exercise techniques on the electromyographic (EMG) activities of the quadriceps femoris muscle have been studied (Cerny, 1995; Hodges and Richardson, 1993; Hanten and Schulthies, 1990, Karst and Willet, 1995; Hanten and Schulthies, 1990; Doucette and Child, 1996; Zakaria et al., 1997). The findings in previous studies did not yield a consensus on an exercise protocol that would be the most effective in strengthening the VMO and the VL muscles. While some studies showed higher EMG activities in the VMO muscle during terminal knee and hip adduction exercises (Cerny, 1995; Hodges and Richardson, 1993; Hanten and Schulthies, 1990), other studies did not find any significant difference in the EMG activities in the VMO muscles during both exercises (Karst and Willet, 1995; Hanten and Schulthies, 1990; Doucette and Child, 1996; Zakaria et al., 1997).

A limitation to the existing studies is that the terminal knee extension and hip adduction exercises were performed isometrically. Given that the exercise method prescribed for patients with PFPS is isotonic in nature, there is the need to compare the EMG activities in the VMO and VL muscles during isotonic exercise therapy. This study was designed to compare the EMG activities in the VMO and VL muscles during hip adduction and terminal knee extension exercises. We hypothesized that (a) the isotonic: hip adduction exercise would produce higher EMG activities in the VMO muscle than the VL muscle, and (b) that the terminal knee extension exercise would not produce higher EMG activities in the VMO muscle compared to the VL muscle.

\section{METHODOLOGY}

\section{Subjects}

The study protocol conformed to the Helsinki declaration principles for the use of human subjects in experimental research. Informed consent was obtained from the subjects prior to data collection. A sample of convenience of 25 (14 females and 11 males) healthy subjects participated in the study. Their ages ranged from 20-45 years $(\mathrm{M}=29.5, \mathrm{SD}=$ 6.8). Volunteers were excluded from the study if they reported any pain in the knee during functional activities or while they are at rest.

\section{Research Design}

The A2 (testing condition) x 2 (exercise type) x 2 (gender) repeated measures quasi-experimental factorial design was utilized in this study. The independent variable included the hip adduction exercise and the terminal knee extension exercise. The dependent variable was the EMG activities monitored by the Orion Model 8600 biofeedback system

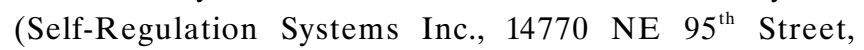
Redmond, Washington 98052).

\section{Procedure}

On report to the laboratory, the subjects were asked which leg they would use to kick a soccer ball. Their response to the question identified their dominant leg. Subsequently, the anterior surface of the knee of the dominant leg was shaved and the skin abraded with alcohol in order to reduce skin impedance. The active electrode of the EMG monitor was placed over the most distal portion of the bellies of the VL and VMO muscles. The reference electrode was placed one inch proximal to the active electrode and the ground electrode placed on the tibia tubercle. The electrodes were held in place with an elastic band.

The first exercise method to be performed was determined randomly by a coin toss and the subjects performed the second exercise method after the EMG readings were recorded after the first trial. Since an EMG machine was used with only one channel monitoring capacity, the toss of a coin was employed to determine the order of selection of the muscle to be tested. To standardize the techniques of the exercises performed, one of the investigators demonstrated the exercise and another investigator gave consistent verbal cues during the testing. The screen of the EMG machine was positioned away from the subject in order to reduce the impact of visual feedback during the testing.

During the hip adduction exercise, the subjects were positioned on their side lying on the dominant leg, with the hip in zero degrees of rotation. The non dominant leg was positioned in 90 degrees of rotation and in 90 degrees of hip flexion on a stool to keep the pelvis neutral and to allow for unobstructed active hip adduction. During the terminal knee extension exercise, the subjects were positioned supine with towel rolls underneath their dominant knee to allow 40 degrees of flexion; because the knee range of motion from 0-40 degrees is where most functional activities are performed and is also where the patella is least stable (Doucette \& Child, 1966). The range of motion measurements during the testing was verified with a universal goniometer. 
Subjects performed one randomly assigned exercise ten times on the dominant leg and the EMG activities were recorded from a randomly selected muscle (VL or VMO). Subjects then performed ten repetitions of the exercise on the same leg and the EMG activities from the second muscle were recorded. The procedure was then repeated in the same way for the second exercise. For each repetition, subjects were instructed to move slowly, hold the contraction for two seconds, relax slowly, and rest for two seconds. The readings for average, high, and low EMG activities were recorded during the ten repetitions of each exercise. The high and low EMG activities were recorded to examine the range of muscle activation, but only the average EMG activities were utilized for statistical analysis.

\section{Statistical Analysis}

An independent t-test was used to determine any differences between the physical characteristics of the male and female participants in the study. A three factor repeated measures analysis of variance (ANOVA) was employed to determine possible differences in the EMG activities in the VMO and VL muscles during hip adduction and terminal knee extension exercises and between genders. The Duncan post hoc test was utilized to examine significant main effects. The level of significance was fixed at $\mathrm{p}<.05$ level. The statistical package for social sciences (SPSS) was used for data analysis (SPSS Inc., 444 N Michigan Ave., Chicago, IL 60611).

\section{RESULTS}

The physical characteristics of the participants are presented in table 1 . The results of the t- test revealed that the male and female participants were similar in age, height and weight.

Table 1. Physical characteristics of participants

\begin{tabular}{ccccc}
\hline \multirow{2}{*}{ Variable } & $\begin{array}{c}\text { Males } \\
(\mathrm{n}=11)\end{array}$ & $\begin{array}{c}\text { Females } \\
(\mathrm{n}=14)\end{array}$ & T-value & P-level \\
\cline { 2 - 3 } & $\mathrm{M}(\mathrm{SD})$ & $\mathrm{M}(\mathrm{SD})$ & & \\
\hline Age (years) & $29.5(5.3)$ & $27.5(5.3)$ & -0.78 & 0.27 \\
Height $(\mathrm{cm})$ & $178.8(5.3)$ & $160.6(6.6)$ & -0.70 & 0.21 \\
Weight $(\mathrm{kg})$ & $87.2(16.4)$ & $59.3(9.8)$ & -0.50 & 0.08 \\
\hline
\end{tabular}

The result of the three-way repeated ANOVA for the EMG activities (table 2) did not show any significant main effects for exercise type and testing conditions but the terminal knee extension exercise type $\mathrm{x}$ gender interaction effect was statistically significant $(\mathrm{F}=8.28, \mathrm{p}<.001)$. The result of the Duncan post-hoc test revealed that the male participants, during terminal knee extension exercise, produced higher $(\mathrm{p}<.05)$ EMG activities in the VL muscle than in the VMO muscle. For both genders, no significant difference was found in the EMG activities of both muscles during the hip adduction exercise. On the contrary, the EMG activities in the VMO and VL muscles were the same during the hip adduction exercise. Females did not demonstrate any significant differences in the EMG activities in their VMO and VL muscles during the two exercise protocols (figure 1).

Table 2. Summary of the analysis of variance of the EMG activities in the vastus medialis oblique and vastus lateralis muscles during hip adduction and terminal knee extension exercise protocols

\begin{tabular}{|c|c|c|c|c|c|c|c|c|}
\hline \multicolumn{3}{|c|}{ Sources of Variance } & DF & \multicolumn{2}{|r|}{ SSQ } & MS & F-ratio & $\mathrm{P}$-values \\
\hline \multicolumn{3}{|c|}{ Hip add } & 1 & \multicolumn{2}{|r|}{25.776} & 25.776 & 0.85 & 0.366 \\
\hline \multicolumn{3}{|c|}{ Error (within groups) } & 24 & \multicolumn{2}{|r|}{729.730} & 30.410 & & \\
\hline \multicolumn{3}{|c|}{ TKE } & 1 & \multicolumn{2}{|r|}{140.450} & 140.45 & 2.16 & 0.155 \\
\hline \multicolumn{3}{|c|}{ Error (within groups) } & 24 & \multicolumn{2}{|r|}{1560.460} & 65.02 & & \\
\hline \multicolumn{3}{|c|}{ Gender } & 1 & \multicolumn{2}{|r|}{1187.610} & 1187.61 & 2.85 & 0.105 \\
\hline \multicolumn{3}{|c|}{ Error (within groups) } & 23 & \multicolumn{2}{|r|}{416.701} & & & \\
\hline \multicolumn{3}{|c|}{ TKE x Gender } & 1 & \multicolumn{2}{|r|}{412.960} & 412.96 & 8.28 & 0.009 \\
\hline \multicolumn{3}{|c|}{ Error (within groups) } & 23 & \multicolumn{2}{|r|}{1147.450} & 49.89 & & \\
\hline \multicolumn{3}{|c|}{ Hip add $x$ Gender } & 1 & \multicolumn{2}{|r|}{22.120} & 22.12 & 0.72 & 0.410 \\
\hline \multicolumn{3}{|c|}{ Error (within groups) } & 23 & \multicolumn{2}{|r|}{707.620} & 30.77 & & \\
\hline DF & $=$ & Degrees of Freedom & & Hip add & Hip & & & \\
\hline SSQ & $=$ & Sum of Squares & & TKE & Tern & ension & & \\
\hline MS & $=$ & Mean of Squares & & Gender & Male & & & \\
\hline
\end{tabular}




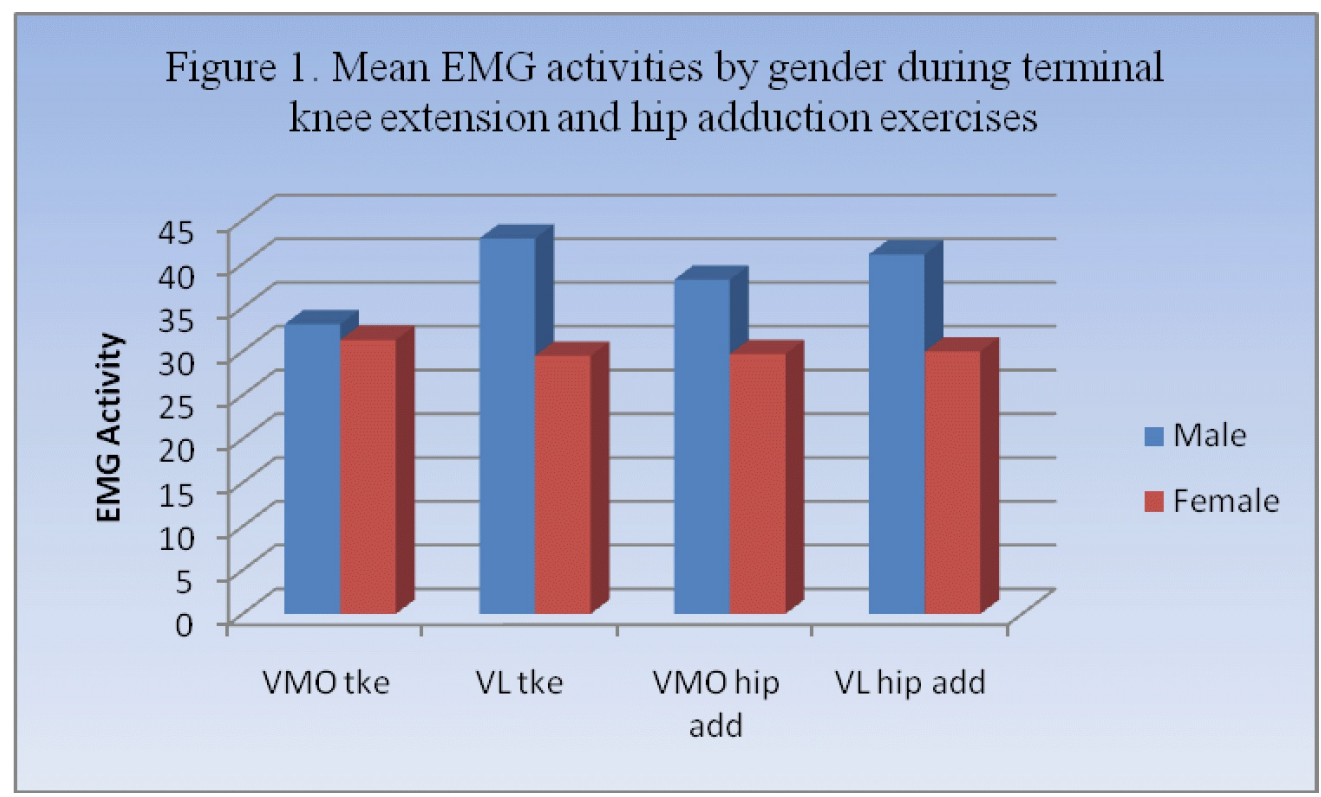

\section{DISCUSSION}

This study was designed to compare in both male and female subjects the EMG activities in the VMO and VL muscles during two open chain exercises used in the management of PFPS. Our findings revealed that among male participants, during the terminal knee extension exercise, the EMG activities in their VL muscle were significantly higher than in the VMO muscle. No significant difference was found for both genders in the EMG activities of both muscles during the hip adduction exercise. For any exercise therapy to be effective in strengthening muscles, it is crucial that it targets the specific muscle group. Our findings did not support our hypothesis that the hip adduction exercise would produce higher EMG activities in the VMO muscle than in the VL muscle during the terminal knee extension exercise.

Our hypothesis was based, in part, on the findings by Hanten and Schulthies (1990) who demonstrated significantly greater EMG activities in the VMO muscle than in the VL muscle during the hip adduction isometric exercise. However, it is pertinent to note that our study design is different from that of Hanton and Schulthies. They did not explore the influence of gender in their study and they used indwelling needle electrodes and the incorporation of medials tibia rotation during isometric hip adduction exercises. Although Hanton and Schulthies (1990) found higher activation of VMO during an isometric exercise, it is important to recognize the practical relevance of the design in this study which utilized an isotonic exercise regime.

The study by Cerny (1995) supports the findings that neither the hip adduction nor the terminal knee extension exercise protocols produced higher EMG activities in both VMO and VL muscles, even when the patella was taped. The study utilized both a healthy population and subjects with PFPS, but there was a large discrepancy between the number of subjects used in each group. The healthy group had twice the number of study participants compared to the patients with PFPS. The study incorporated the use of open and closed chain exercises.

The finding by Hodges and Richardson (1993) is consistent with this study which showed that the EMG activities in the VMO and VL muscles are not significantly different during the non-weight-bearing hip adduction exercise. In the study by Hodges and Richardson, the EMG activities in the VMO muscle increased relatively more than in the VL muscle during the hip adduction exercise performed in the weight-bearing posture. Their finding supports the use of functional weight-bearing exercises as opposed to straight-plane open-chain exercises, to maximize the activation of the VMO muscle.

The present study had limitations that affected the external validity of the findings. One of these is the use of a sample of convenience with a healthy population; the findings therefore, may not be generalized to patients with PFPS. Also, though the surface electrode used in this study is considered an acceptable measure of muscle 
activity, it is not as precise as indwelling needle electrodes, due to the 'cross-talk' phenomenon. The fact that only a one channel EMG machine was used is another limitation, because only the activities in the VMO or the VL could be monitored for each exercise protocol.

From a preventive standpoint, PFPS can be relieved by avoiding activities that make the symptoms worse. Patients should be told to avoid sitting or kneeling in the bent-knee position for long periods and to avoid bent-knee exercises, such as squats, deep knee bends, or 90-degree leg extensions. Cyclists, should be advised to adjust a bicycle so that the resistance is not too great and the seat is at an appropriate height. The rider should be able to spin the pedals of the bicycle without shifting weight from side to side, and the legs should not be fully extended at the lowest part of the pedal stroke.

While there are other conservative treatments prescribed for patients with PFPS such as knee kinesiotaping techniques and the use of a brace to stabilize the patella, further studies are needed to identify the exercise method that would reduce the hypothesized imbalances in strength and timing during recruitment of the VL and VMO muscles. Further research that involves common exercise therapies such as isotonic and functional weight bearing activities, should be examined to determine their effect on the VMO muscle. For relevance, it is important to investigate the effects of the various exercise therapies on patients with patellofemoral pain.

\section{CONCLUSION}

It was found in the study that among male participants during the terminal knee extension exercise the EMG activities in the VL muscle were significantly higher than in the VMO muscle. Follow-up studies are needed to determine the most effective exercise strategy for strengthening the quadriceps femoris muscle in both males and females.

\section{References}

Cerny, K. 1995. Vastus medialis oblique/vastus lateralis muscle activity ratios for selected exercises in persons with and without patellofemoral pain syndrome. Phys Ther 75(8): 672683.

Chondromalacia patellae.

http://www.jgames.co.uk/title/Chondromalacia_patellae. Retrieved on August 26, 2009.
Chondromalacia Patella. SportsMed http://www.medicinenet.com/patellofemoral_syndrome/art icle.htm. Retrieved on August 5, 2009.

Doucette, S.A., D.D. Child. 1997. The effect of open and closed chain exercise and knee joint position on patellar tracking in lateral patellar compression syndrome. J Orthop Sports Phys Ther 23(2): 104-110.

Grelsamer R. P. Patellar Nomenclature 2005. The Tower of Babel Revisited. Symposium on Patellofemoral Arthroplasty. Clin. Orthop 436:60-65.

Hanten, W.P. and S.S. Schulthies. 1990. Exercise effect on electromyographic activity of the vastus medialis oblique and vastus lateralis muscles. Phys Ther 70(9): 561-565.

Hecox, B., T.A. Mehreteab, J. Weisberg and J. Sanko. 2006. Integrating Physical Agents in Rehabilitation. Second Edition. Pearson Practice Hall, Upper Saddle River, NJ.

Hodges, P.W., C.A. Richardson. 1993. The influence of isometric hip adduction on quadriceps femoris activity. Scand $J$ Rehabil Med 25(2): 57-62.

Jenkins M A , C. Honig. 2005. Patello-Femoral Syndrome. (http://www.rice.edu/ jenky/sports/pfs.html. Retrieved August 6, 2009.

Karst, G.M., G.M. Willett 1995. Onset timing of electromyographic activity in the vastus medialis oblique and vastus lateralis muscles in subjects with and without patellofemoral pain syndrome. Phys Ther 75(9): 813-823.

Patellofemoral pain syndrome: Topic overview. WebMD Medical Reference from Healthwise (http://www.webmd.com/a-to-zguides/patellofemoral-pain-syndrome-topic-overview). Retrieved on August 26, 2009.

Schindler, Oliver.2004. Synovial plicae of the knee. Science Direct.

http://www.sciencedirect.com/science?_ob=ArticleURL\& _ $\mathrm{u}$ d i $=$ B 6 W D $9-4 \overline{\mathrm{C}}$ F 15 P 3 1 \&_user $=10 \& \_r d o c=1 \& \_f m t=\&$ orig $=$ search\&_sort $=\mathrm{d} \&$ view $=c \& \_a c c t=C 000050221 \&$ _version $=1 \& \_$urlVersion $=0$ \&_userid=10\&md5 =20253ef5a8701aaa3a975e1dfd63c515. Retrieved on August 15, 2009.

Voight, M.L., D.L.Wieder.1999. Comparative reflex response times of vastus medialis obliquus and vastus lateralis in normal subjects and subjects with extensor mechanism dysfunction: An electromyographic study. Am J Sports Med 19(2): 131-137.

Zakaria, D., K.L Harburn and J.F. Kramer. 1997. Preferential activation of the vastus medialis oblique, vastus lateralis, and hip adductor muscles during isometric exercises in females. J Orthop Sports Phys Ther 26(1), 23-28. 